\title{
LA MISIÓN CRISTIANA EN LA MODERNIDAD LÍQUIDA Una pluralidad necesaria
}

DOI: https://doi.org/10.52039/seminarios.v63i221.60

ARNAUD JOIN-LAMBERT*

Cada semana que pasa muestra la pertinencia de los análisis bastante negativos del sociólogo Zygmunt Bauman sobre la evolución de la sociedad occidental. La metáfora de la liquidez, que ha definido el cambio de milenio, se impone progresivamente como una de las más significativas para explicar la desestructuración y la pérdida de legitimidad de las instituciones en un contexto de triunfo del individuo ${ }^{1}$. El desarrollo en algunas partes de nuevas modalidades de compromiso que Bauman no tiene en cuenta, no invalida todavía su visión. Y como el neo-liberalismo explota y desarrolla el individualismo, las personas se muestran cada vez más fragilizadas. El repliegue sobre sí mismo y el miedo frente al otro prosperan, impulsados por la precariedad social, las consecuencias del calentamiento climático y, de manera más evidente todavía, la crisis de los refugiados y el terrorismo islámico. Los desafíos son múltiples y complejos. Cada grupo, estructurado o no, está llamado a una transformación para hacer frente a las mutaciones, con la amenaza de la desaparición o al menos de perder toda la eficiencia, o degenerar en un comunitarismo sectario. Esta trasformación consiste, según la metáfora de Bauman, en pasar del estado sólido al estado líquido².

* Teólogo católico francés. Es profesor de la Universidad Católica de Lovaina en Bélgica. Enseña teología práctica y liturgia. Actualmente investiga sobre la sinodalidad en las Iglesias particulares. Ha sido presidente de la Société Internationale de Théologie Pratique entre 2014 y 2016.

1. Z. Bauman, L'amour liquide. De la fragilité des liens entre les hommes, Rodez 2004; La vie liquide, Rodez 2006; Le présent liquide. Peurs sociales et obsessions sécuritaires, Paris 2007.

2. En el año 2015 ya me interrogué sobre lo que esto podía significar para la Iglesia católica, cf. mi artículo Hacia una Iglesia «líquida»: Seminarios 217 (2016) 107-117. Las numerosas reacciones a esta perspectiva de la Iglesia líquida han abierto muchos campos de reflexión en las diócesis, los movimientos, los servicios y las comunidades francófonas del primer mundo. 
¿Qué hacer para continuar viviendo en la Iglesia y anunciar el Evangelio hoy? ¿Cómo desarrollar una acción en respuesta al imperativo de Jesús a sus discípulos: «ld y haced discípulos a todas las naciones» (Mt 28, 19)? Dicho con otras palabras, ¿existe un tipo de misión adaptado a una Iglesia líquida en la modernidad líquida?

La cuestión fundamental no estriba tanto en la estructura eclesial como en la manera de realizar la misión. El misionero protestante sudafricano David Bosch habla de "modelo misionero» ${ }^{3}$ como una forma particular de pensar la misión y de integrar la dimensión contextual. Pueden coexistir distintos modelos en un mismo contexto en función de las opciones de los responsables y sus opciones teológicas.

En este artículo examinaremos tres modelos misioneros contemporáneos y un cuarto modelo iniciado por la acción y los discursos del papa Francisco. Mi atención se centrará en su carácter más o menos apropiado a la modernidad líquida. Después, abordaré algunas perspectivas ligadas a la compatibilidad de estos modelos para evaluar la posibilidad misma de una misión cristiana.

Si la cuestión es crucial y decisiva para el futuro del cristianismo, ella no es sin embargo totalmente nueva, sino que ya se planteó en los primeros siglos, antes de la cristiandad, en el reencuentro con el helenismo y la civilización romana.

Previendo este declive de la cristiandad, el papa Juan XXIII planteó el desafío ante los obispos del mundo entero en 1961. A su vez, el concilio Vaticano Il intentó formular la cuestión, que apenas era viva por aquel entonces, ya que consideraba que afecta de lleno a la naturaleza misma de la Iglesia, que es misionera. Ya el 22 de septiembre de 1965, el teólogo dominico Marie-Dominique Chenu pronuncia en una conferencia estas palabras para suscitar la reflexión:

Es de la naturaleza, del ser mismo de la Iglesia, situarse en el mundo; su definición está unida a «la misión», al envío por Cristo, y el término de este envío es su razón de ser; más todavía, es el dinamismo existencial de la Iglesia [...]. Se trata del misterio de la Encarnación, del Dios hecho hombre que ha entrado en la historia y que, continuando de siglo en siglo por y en la Iglesia, determina su ser ${ }^{4}$.

3. D. Bosch, Dynamique de la mission chrétienne. Histoire et avenir des modèles missionnaires, Paris 1995.

4. M. D. Chenu, Une constitution pastorale de l'Église: Documentation hollandaise du Concile 205 (1965) 2; cf. A. Join-Lambert, Annoncer le Christ et l'Évangile: un impératif expérientiel pour le chrétien: Communio 32/5-6 (2007) 127-144. 
TRES NUEVOS MODELOS MISIONEROS IDENTIFICADOS EN EUROPA OCCIDENTAL

Asumiendo el riesgo de ser muy esquemático, distinguimos sintéticamente tres nuevas maneras teóricas de anunciar el Evangelio hoy: la nueva evangelización, la propuesta de la fe y la pastoral «de engendramiento». Como se ve, no consideramos aquí dos opciones por entender que son del pasado: la primera, que puede considerarse la actitud típica de toda dinámica de restauración, pretende mantener a cualquier precio aquello que ha caracterizado durante los siglos de cristiandad a los medios católicos tradicionales y a los tradicionalistas; se trata de una mezcla de repliegue sobre uno mismo y de negación nostálgica, que parece alejada del estilo del Jesús transmitido en los Evangelios por renunciar a la llamada de la misión que tiene en cuenta a todos y se dirige a todos. La segunda, de tono más espiritualista, se resume en la noción de «huida del mundo», que tuvo su apogeo en los años 19501970 , si bien todavía se aprecia en determinadas comunidades religiosas y cristianas, aunque resulta difícil de teorizar.

1. La nueva evangelización es una noción extendida rápidamente en la Iglesia católica, gracias a las exhortaciones del papa Juan Pablo II a partir de 1983. Con ella se expresa la necesidad de realizar de nuevo una evangelización que creíamos ya terminada en los países que eran cristianos. La insistencia del Papa se ha dirigido regularmente a la dimensión de renovación interior, condición para evangelizar a los no creyentes. Esta nueva evangelización es una noción suficientemente compleja para recurrir a la vez a la innovación radical y al recurso de los métodos tradicionales de la misión ${ }^{5}$. Ella se ha realizado principalmente en los movimientos y, de manera especial, en las nuevas comunidades, mostrando en profundidad la gran dificultad de las parroquias a dejar su modo estático, y por eso "sólido» del anuncio del Evangelio. Así resuenan las palabras del papa Francisco en 2013: "La pastoral en términos misioneros exige abandonar el confortable criterio pastoral de 'siempre se ha hecho así'. Yo invito a cada uno a ser audaz y creativo en este deber de repensar los objetivos, las estructuras, el estilo y los métodos de evangelización de sus propias comunidades ${ }^{6}$.

La dinámica característica de las prácticas misioneras que reclama la nueva evangelización es un «ir hacia». Se trata de encontrar los medios y los métodos para entrar en relación con los alejados de la Iglesia, sean

5. Cf. el interesante estudio de J. Rigal, La nouvelle évangélisation. Comprendre cette nouvelle approche. Les questions qu'elle suscite: Nouvelle Revue de Théologie 127 (2005) 436-454.

6. Francisco, Exhortación apostólica Evangelii gaudium, 33. 
católicos o no, creyentes o no. El objetivo último es su ingreso o retorno a la Iglesia, pensada como comunidad de salvación. Los ejemplos serían los cursos Alpha para los que están más cerca, las misiones urbanas de la comunidad Emmanuel para el ámbito local $^{7}$ y las Jornadas Mundiales de la Juventud como evento de gran alcance. Las cuestiones que se plantean normalmente a este modelo misionero son las articulaciones entre emoción y reflexión, entre tiempos fuertes e inserción continuada en una comunidad, entre el grupo y la identidad bien afirmada y la apertura a la alteridad, incluso intra-eclesial.

2. El segundo modelo misionero es el fruto de una proceso consultivo y reflexivo de muchos años bajo la dirección del obispo Claude Dagens. Los obispos franceses lo han adoptado con el nombre de "proposición de la fe». La Carta a los católicos de Francia de 1996 es un documento conclusivo que refleja las preocupaciones pastorales de finales de la década de 1980, una carta propositiva que busca anunciar el Evangelio en un contexto que ya no es el de la cristiandad ${ }^{8}$. Los obispos de Francia han hecho aquí de pioneros, seguidos después por casi todos los episcopados de Europa oriental. Ellos invitan a «reencontrar el gesto inicial de la evangelización: aquel de la proposición simple y decidida del Evangelio». Para esto, en primer lugar se trata de ir al corazón de la fe, lo que es similar a la nueva evangelización, para realizar un trabajo de discernimiento. La gran diferencia con la nueva evangelización reside en el lugar de este anuncio, que es la práctica ordinaria de la Iglesia, sobre todo los encuentros pastorales que pueden surgir cuando las personas alejadas de la comunidad vienen a solicitar estas prácticas de fe, sin importar sus motivaciones. No hay un método específico. El acento está en aquello que es el centro de la relación en ese momento, inscribiendo por eso el anuncio del Evangelio en un proceso antes que en un acontecimiento. Los obispos insisten también sobre la pertinencia social de la proposición de la fe. Hay un valor añadido en el Evangelio que sirve para vivir juntos en una sociedad plural que evoluciona. A diferencia de la nueva evangelización, la clave está en la calidad de la acogida: no tanto en el «ir hacia» como en el «recibir».

7. Cf. W. Hagemann - B. Leahy, «Seht, wie sie einander lieben!» Die Konsequenzen des marianischen Profils in den Neuen Geistlichen Gemeinsachaften für Kirche, Gemeinde und Gesellschaft, en Ch. Hegge (dir.), Kirche bricht auf. Die Dynamik der Neuen Geistlichen Gemeinschaften, Münster 2005, 178-195, un original artículo sobre los modelos misioneros que animan las nuevas comunidades: Emmanuel, San Egidio, Focolares, Fraternidades monásticas de Jerusalén.

8. Les Évêques de France, Proposer la foi dans la société actuelle. Lettre aux catholiques de France, Paris 1996. 
3. El tercer modelo misionero es la denominada pastoral del «engendramiento». Su origen es muy diferente de los dos anteriores. Su teorización se basa, por una parte, en observaciones de prácticas y testimonios de actores sobre el terreno, y de otra, en reflexiones de algunos teólogos ${ }^{9}$. Una convicción común surge de estas prácticas y sirve de reflexión: «El Evangelio actúa en las conciencias de hoy como en las de ayer». Se considera la fe como un germen que está en el otro y no como una cosa que se le tenga que transmitir. La misión no es tan asimétrica, o sea, de alguien que la tiene o que conoce hacia quien no conoce nada. Lo importante es la calidad de la relación, del encuentro, donde cada persona aporta alguna cosa. La noción de hospitalidad podría resumir bien la clave de este modelo misionero. Gracias a la riqueza de la palabra, cada uno es huésped del otro, Cristo mismo se hace hoy huésped como ha sido desde siempre. Entre los medios privilegiados y a privilegiar es fundamental la Biblia, recibida como Palabra de Dios. Los lugares de desarrollo de una pastoral de este tipo son los pequeños grupos con una proximidad social o geográfica. La pastoral del engendramiento es marginal cuantitativamente y bastante «francesa», con buena acogida en Bélgica, un poco en Suiza e inesperadamente en Alemania.

\section{¿UN CUARTO MODELO MISIONERO POR INICIATIVA DEL PAPA FRANCISCO?}

Cuando el papa Benedicto XVI adoptó la expresión conocida de nueva evangelización, cambió su manera de considerar la misión, para equilibrar lo que sería a veces inestable y no teniendo en cuenta el lugar del anuncio. Su propio modelo misionero resultaría paradójicamente más asertivo y dialogante que el de Juan Pablo II. Queriendo unir los modos y la finalidad de la misión, el Ratzinger ha propuesto el vocabulario de «atrio» y promovido los «atrios de los gentiles». Si esta variante de la nueva evangelización se sitúa «fuera» para "aquellos de fuera», se sitúa también con la finalidad de hacerlos entrar. El «atrio» no es más que un lugar de transición que no tiene finalidad propia. El movimiento fundamental es el mismo que la nueva evangelización original.

El papa Francisco ha modificado esto imponiendo una dinámica de salida, que no considera el retorno como primer objetivo. Esto justifica ya hablar de un cuarto modelo misionero. El discurso del cardenal Bergoglio en

9. P. Bacq - Ch. Theobald (dir.), Une nouvelle chance pour l'évangile: vers une pastorale d'engendrement, Paris 2004; también, Passeurs d'Évangile. Autour d'une pastorale d'engendrement, 2006. Cf a este respecto la profuesta que hace Theobald en su libro El estilo de la vida cristiana, Sígueme, Salamanca 2016, 131-151. 
el cónclave de 2013 no tenía ambigüedad: «La Iglesia está llamada a salir de ella misma y a ir a las periferias, las periferias geográficas, pero igualmente existenciales: allí donde reside el misterio del pecado, el dolor, la injusticia, la ignorancia, allí donde lo religioso, el pensamiento, son despreciados, allí donde están todas las miserias ${ }^{10}$. Tras su elección papal, no ha dejado de poner en obra este programa, que consiste más en un cambio fundamental de actitud que en recetas concretas. Si Francisco emplea aún los términos «nueva evangelización», es únicamente para situarse en continuidad con sus predecesores (Evangelii gaudium 11, por ejemplo). De hecho, prefiere claramente el vocabulario misionero, que no puede dejar las cosas como están ${ }^{11}$. La finalidad de la misión es el otro por sí mismo. El Papa invita a entrar en una postura de diálogo, dando al otro todo el precio que tiene a sus ojos y a los ojos de Dios. Nosotros llamaremos a este modelo «Iglesia en salida», expresión recurrente en Francisco ${ }^{12}$. Por mi parte, propongo caracterizar su finalidad como la formación de una comunidad humana de salvación.

Examinando los propósitos incesantes del papa Francisco sobre la misión y la actitud del «discípulo-misionero», parece que nos hallamos ante un modelo misionero diferente a los tres anteriores. Solamente es posible comprenderlo a partir del dinamismo principal del movimiento de relación de la Iglesia al «otro de fuera de la Iglesia», y según el iniciador de la misión. La «nueva evangelización» (entendida en el sentido de Juan Pablo II o en el de Benedicto $\mathrm{XVI}$ ) representa una iniciativa unilateral de la Iglesia, que va desde lo interno hacia lo externo y establece una relación asimétrica con «el otro». La "proposición de la fe» sería una iniciativa bilateral, que va principalmente desde lo externo hacia lo interno, de tal modo que la relación permanece más bien asimétrica, porque «el otro» pide y la Iglesia le propone caminar más lejos que su necesidad expresada. La pastoral del «engendramiento» sería una iniciativa unilateral de los cristianos ligados más o menos de forma explícita con la institución, en un movimiento de diálogo y estableciendo localmente una relación simétrica. Esto se ve en el cuadro siguiente:

10. Card. J. Bergoglio, Discurso al cónclave 2013, reconstruido por él mismo según sus propias notas.

11. Papa Francisco, Evangelii gaudium, 25.

12. Por ejemplo, en su Discurso a la 28 asamblea plenaria del Consejo pontificio para los laicos, 17 de junio de 2016: «Quisiera proponeros como horizonte de referencia para vuestro futuro inmediato, un binomio que se podría formular así: Iglesia en salida - laicado en salida». 
Misión cristiana y modernidad líquida

\begin{tabular}{|c|c|c|c|c|}
\cline { 2 - 5 } \multicolumn{1}{c|}{} & $\begin{array}{c}\text { Nueva } \\
\text { evangelización }\end{array}$ & $\begin{array}{c}\text { Proposición } \\
\text { de la fe }\end{array}$ & $\begin{array}{c}\text { Pastoral de } \\
\text { engendramiento }\end{array}$ & $\begin{array}{c}\text { lglesia } \\
\text { en salida }\end{array}$ \\
\hline $\begin{array}{c}\text { Origen de } \\
\text { la iniciativa }\end{array}$ & $\begin{array}{c}\text { unilateral (pro- } \\
\text { yecto pastoral) }\end{array}$ & bilateral & $\begin{array}{c}\text { unilateral (de } \\
\text { comunidades) }\end{array}$ & $\begin{array}{c}\text { unilateral (pro- } \\
\text { yecto pastoral) }\end{array}$ \\
\hline Dinámica & $\begin{array}{c}\text { de lo interno } \\
\text { a lo externo }\end{array}$ & $\begin{array}{c}\text { de lo externo } \\
\text { a lo interno }\end{array}$ & dialógica & dialógica \\
\hline Finalidad & $\begin{array}{c}\text { retorno a lo } \\
\text { interno } \\
\text { retorno a lo } \\
\text { interno }\end{array}$ & $\begin{array}{c}\text { co-construcción } \\
\text { de sentido }\end{array}$ & $\begin{array}{c}\text { formar una co- } \\
\text { munidad huma- } \\
\text { na de salvación }\end{array}$ \\
\hline $\begin{array}{c}\text { Relación } \\
\text { Iglesia- } \\
\text { mundo }\end{array}$ & asimétrica & asimétrica & simétrica & simétrica \\
\hline
\end{tabular}

Porque estos modelos no tienen la misma génesis ni el mismo desarroIlo, podrían existir otros, con algunas variantes o radicalmente diferentes, en todo caso pertinentes en otros contextos locales o regionales. $Y$ otros modelos podrían todavía aparecer. Por nuestra parte, nos remitimos a estos cuatro modelos.

\section{¿INCOMPATIBILIDAD DE MODELOS MISIONEROS?}

El cuadro, necesariamente esquemático, muestra la delicada cuestión de la compatibilidad entre los modelos misioneros. Antes de la llegada del papa Francisco, los tres primeras columnas ayudan a releer y a comprender los interminables debates o conflictos entre los católicos comprometidos con su fe, también entre las comunidades parroquiales y las comunidades religiosas tradicionales o nuevas. La aparición de este modelo de Iglesia en salida, de Iglesia como «hospital de campaña» (por citar una imagen fuerte del papa Francisco) no podía volver más incierto el fundamento de cada uno de los modelos misioneros desarrollados. Los actores y practicantes de la nueva evangelización fueron y están sorprendidos por esta manera de anunciar, que es menos asertiva y más empática. Por otra parte, las personas entusiasmadas por la pastoral de engendramiento sienten y expresan una afinidad con las palabras del papa Francisco. Es probablemente debido a la dinámica dialogal, promovida en uno y otro modelo, y en la relación Iglesia/mundo simétrica. Sin embargo, la dimensión fuertemente institucional del modelo del papa Francisco se debate en estos grupos y comunidades.

Estos modelos misioneros aparecen a primera vista como incompatibles o, al menos, difícilmente compatibles. ¿Y para qué sirven? En primer lugar, hay que señalar que no se trata de una historia de expertos. El papa Francis- 
co afirma con fuerza: «Todos los cristianos son constituidos misioneros» ${ }^{13}$. Cada uno, examinándose, se siente de manera espontánea más cerca de uno de los cuatro modelos. Y mirando el cuadro vemos el modelo misionero que aceptaríamos o al menos promoveríamos. Y esto toca a la intimidad de cada uno. Cómo anunciar este Cristo en el que creo y que es más íntimo de mí que yo mismo (por parafrasear a san Agustín). Las diferencias de apreciación sobre el modelo misionero tienen entonces sus raíces más profundas en cada persona. Como esto forma parte de nuestra relación con el mundo y con el otro, comprendemos fácilmente que estos modelos son incompatibles a nivel del individuo. "Yo» no puedo asumir dos modelos al mismo tiempo. ¡ Yo» no puedo ser san Pedro y san Pablo al mismo tiempo! Sólo puede entonces cambiarse gracias a una transformación interior, es decir, a una conversión. Por una experiencia personal, yo descubro que este modelo es más apropiado para mí hoy. Los sacerdotes interrogados en mis investigaciones sienten que deben diversificar los estilos de reencuentro y las prácticas pastorales, pero para ellos no es tan fácil asumir opciones diferentes.

La importancia de la autenticidad es una dimensión clave de la modernidad líquida que sirve para evaluar una autoridad. Una persona debe ser creíble para que su discurso sea acogido. En todo anuncio del Evangelio hay entonces una exigencia importante de que las palabras y gestos estén arraigadas en la existencia. No cabe duda de que la fe es un camino que manifiesta modos de ser y palabras diferentes según las edades de la vida. El camino se inicia con una complejización creciente para luego simplificarse poco a poco. El hecho de no fijarse definitivamente en las palabras y actitudes está en la línea de los rasgos de la modernidad líquida. Algunos teólogos como Christoph Theobald, han mostrado cómo esto se ve también en el Evangelio ${ }^{14}$. Hay así muchas maneras de vivir la fe cristiana en la tradición católica latina, que se expresan con actitudes diversas y palabras diferentes.

Pero si los diferentes modelos misioneros no son compatibles, ¿pueden acaso ser complementarios? La cuestión puede parecer paradójica, pero no carece de importancia. Si les situamos al lado de los destinatarios de la misión-aquellos y aquellas que los cristianos reencuentran en lo cotidiano o en ámbitos excepcionales- es razonable pensar que todos no son sensibles al mismo modo relacional ni al mismo tipo de discurso sobre las cuestiones de sentido. Y la experiencia lo confirma. Algunas personas son alcanzadas por un anuncio directo e inesperado de la palabra; otros por la calidad del

13. Papa Francisco, Mensaje para la 54 Jornada mundial de oración por las vocaciones 2017, 27 de noviembre de 2016.

14. Cf. sus importantes volúmenes Le christianisme comme style. Une manière de faire de la théologie en postmodernité, Paris 2007. 
caminar vivido oportunamente en una demanda dirigida a una comunidad cristiana; otros al compartir, en amistad y cercanía, las cuestiones existenciales; otros en el reencuentro de cristianos que les manifiestan el gran valor que ellos tienen a los ojos de Dios y de la Iglesia. Es imposible decir si estas mismas personas habrían sido alcanzadas por una iniciativa diferente de los cristianos. En todo caso, la pluralidad de modelos misioneros es un hecho admitido. Los responsables eclesiales y los cristianos de hoy deben tenerlo en cuenta. Todos estos modelos son al menos complementarios para anunciar el cristianismo por distintos caminos.

\section{ADECUACIÓN DE LA MISIÓN AL CONTEXTO DE LA MODERNIDAD LÍQUIDA}

¿Esta complementariedad pragmática debe hacernos renunciar a tener una opinión sobre la adecuación o no de diversos modelos misioneros en la modernidad líquida? ¿Acaso vale todo? Y la misión, sin importar cómo, ¿puede seguir mientras el Evangelio sea anunciado?

Resulta delicado responder a estas preguntas. Para ello conviene distinguir entre los individuos y las comunidades. Hasta el momento presente el individuo era el objetivo de la reflexión, pero ahora le corresponde a la Iglesia, que es por esencia misionera, según recordaba la víspera de la clausura del concilio Vaticano II el padre Chenu. Nos preguntamos cómo la Iglesia líquida, que se perfila bajo el modelo complexivo de la modernidad líquida, puede ser misionera en este contexto.

La primera respuesta sería decir que «toda» la Iglesia debe volverse una «Iglesia en salida», siguiendo las modalidades concretas para poner en obra las orientaciones reiteradas por el papa Francisco. Además de lo que sería un «argumento de autoridad» del Papa, parece que la dinámica dialógica es la única que funciona en la modernidad líquida. Según las obras de Bauman, una sociedad de este tipo rechaza las posturas asimétricas o, como mucho, las acoge con desconfianza. La nueva evangelización y la proposición de la fe encuentran aquí sus límites. Al contrario, hay una buena adecuación entre la «Iglesia en salida» del papa Francisco y las expectativas de nuestros contemporáneos - por lo menos occidentales-en el ámbito de las cuestiones de sentido. El Evangelio podría entonces ser percibido como un mensaje existencial, trascendente y positivo para cada individuo y para todos.

Esta opción preferente es sin embargo simplista si se la considera única y excluyente de las otras. Cada modelo misionero no es aplicable en todas partes y por todos. La realidad resiste a toda uniformización. Yo propongo, pues, considerar otra característica de la modernidad líquida, aquella de la ausencia de valores y las afiliaciones parciales y temporales de los individuos 
a los grupos. Lo propio de una Iglesia líquida consiste en no adoptar un modelo misionero «único», eficaz en la modernidad líquida, aunque este modelo haya sido promovido por el Papa. La Iglesia sólo sería líquida desde el punto de vista de la misión, permitiendo y al mismo tiempo incitando a la coexistencia de muchos modelos misioneros en cada Iglesia particular (diócesis). Por decirlo de otra forma, una diócesis debe asumir en ella una pluralidad misionera. Esta necesidad se impone también, pero de manera diferente, en las grandes órdenes y congregaciones religiosas. Es aquí donde podemos hablar, como el papa Francisco, de conversión misionera. Las diócesis europeas han realizado en su mayoría elecciones pastorales univocas, tanto para la catequesis como para sus estructuras o sus opciones diaconales, etc. Esto se observa muy claramente en las decisiones salidas de los sínodos diocesanos y en otras asambleas desde hace treinta años. Ese tiempo ha pasado, pues experimenta con dolor que no puede responder a la urgencia misionera propia del cristianismo. Viene la hora en la que el trabajo de los responsables diocesanos tendrá que organizar la pluralidad, discerniendo la validez de sus acciones en función de los bautizados, entendidos como agentes de la misión, y las mujeres y hombres que están a su alrededor.

Una cuestión sigue abierta: el «lugar» desde el que debe organizarse una pluralidad de la misión. Las reagrupaciones de las parroquias en Europa han dado lugar a unidades pastorales que cuentan con muchas comunidades, sacerdotes y laicos con responsabilidades. Este hecho cambia, sin duda, los límites de las antiguas parroquias multiseculares. Y aquí se juegan la conversión misionera y la pluralidad de modelos. Además, es ahí donde que puede desplegarse una Iglesia líquida articulando muchos polos y tipos de presencia en el mundo ${ }^{15}$.

La postura de diálogo del modelo de Iglesia en salida parece el modelo misionero que debemos privilegiar. Se trata, en efecto, de la única actitud que permite desarrollar una pluralidad también interna en la Iglesia. Esto vale para todos los ámbitos de la pastoral. Sabemos que los obispos son los primeros afectados y deben tomar distancia con el modelo misionero en el que se sienten mejor. Ellos deben aprender aquello en lo que no están formados. Y no hay nada más difícil para una organización que estructurar una diversidad de opciones y de acciones. Hace falta organizar, en la teoría y en la práctica, esta nueva manera de ver. ¡Frente al suicidio institucional hay que renovarse! Esto explica las tentaciones puntuales de repliegue hacia las ideas restauradoras de una cristiandad desaparecida. En todo caso, la urgencia no debe conducir a la improvisación, sino a formar los agentes

15. Cf. la noción «hospital líquido» en A. Join-Lambert, Hacia una Iglesia «líquida». 
pastorales, sacerdotes y laicos, para organizar esta pluralidad también localmente. En el ámbito de la formación hoy se debe construir todo o casi todo.

La conversión misionera de una Iglesia particular va a consistir en repensar su propia estructura a partir de la finalidad misionera y los modos operativos. La Iglesia logrará entonces adaptarse a su contexto, siguiendo así su misión bimilenaria. El desplazamiento exigido tiene en cuenta el horizonte hacia el cual se debe tender: la humanidad sufriente en todas sus dimensiones, incluyendo la espiritual, pues el Evangelio puede dar respuesta a la falta de esperanza de la sociedad.

¿Cómo realizar en concreto esta conversión? Aparentemente, el Papa parecía proponer un modelo misionero preferente. ¿En detrimento de los otros? La hipótesis de este artículo es la necesidad de una pluralidad en la organización y la misión. ¿Cómo pensarla y vivirla tanto a escala universal como localmente? La Iglesia tiene recursos inestimables como la sinodalidad (inseparable de la colegialidad episcopal y de la primacía del pontificado ${ }^{16}$ ). El papa Francisco, en un discurso para la reflexión, ha afirmado: «El camino de la sinodalidad es justamente esto que Dios espera de la Iglesia del tercer milenio ${ }^{17}$. Él mismo, en otra serie de discursos que abordan este tema, exhorta recurrentemente a hacer más efectiva esta dimensión sinodal en la Iglesia. Así, «una Iglesia sinodal es una Iglesia de la escucha, con la conciencia de que escuchar 'es más que oír'. Es una escucha recíproca en la cual cada uno tiene una cosa que aprender ${ }^{18}$. Encontramos en esta definición la dimensión del diálogo y la simétrica del modelo misionero de la Iglesia en salida. La elaboración y la organización se hacen tanto en los consejos estables como en las asambleas puntuales. $Y$ actuando de manera sinodal en su seno. Y por eso mismo, siempre a la escucha de lo que el Espíritu Santo dice a las Iglesias, según la expresión del libro del Apocalipsis. Cada Iglesia particular y la Iglesia universal encontrarán de ese modo nuevos caminos para recorrer con los hombres y mujeres del tiempo presente. Esta escucha permitiría la coexistencia de modelos misioneros que son poco compatibles a escala individual, condición que es esencial hoy para una fecundidad de la misión cristiana en Occidente.

16. A. Join-Lambert, Synodes et concile en France. Bilan et perspectives, Documents Épiscopat 5, Préface de Mgr L. Ulrich, Conférence des Évêques de France, Paris 2016.

17. Papa Francisco, Discurso en la conmemoración del 50 aniversario de la institución del sínodo de los obispos, 17 de octubre de 2015.

18. Ibid. 